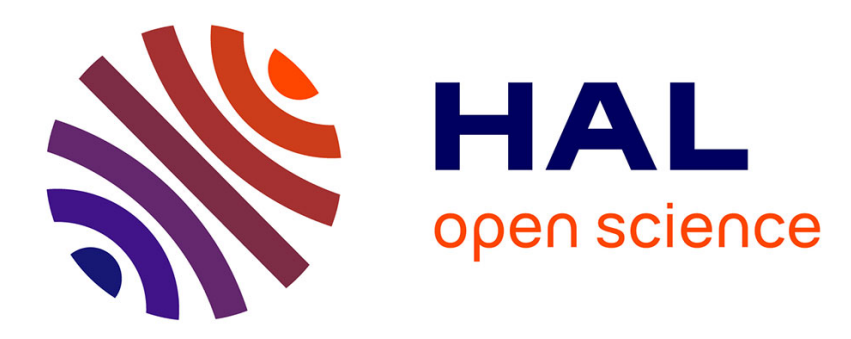

\title{
On numerical construction of homogeneous Lyapunov functions
}

Denis Efimov, Rosane Ushirobira, Jaime A. Moreno, Wilfrid Perruquetti

\section{To cite this version:}

Denis Efimov, Rosane Ushirobira, Jaime A. Moreno, Wilfrid Perruquetti. On numerical construction of homogeneous Lyapunov functions. 56th IEEE Conference on Decision and Control (CDC), Dec 2017, Melbourne, Australia. hal-01611979

\section{HAL Id: hal-01611979 \\ https://hal.inria.fr/hal-01611979}

Submitted on 6 Oct 2017

HAL is a multi-disciplinary open access archive for the deposit and dissemination of scientific research documents, whether they are published or not. The documents may come from teaching and research institutions in France or abroad, or from public or private research centers.
L'archive ouverte pluridisciplinaire HAL, est destinée au dépôt et à la diffusion de documents scientifiques de niveau recherche, publiés ou non, émanant des établissements d'enseignement et de recherche français ou étrangers, des laboratoires publics ou privés. 


\title{
On numerical construction of homogeneous Lyapunov functions
}

\author{
D. Efimov, R. Ushirobira, J.A. Moreno, W. Perruquetti
}

\begin{abstract}
The problem of synthesis of a homogeneous Lyapunov function for an asymptotically stable homogeneous system is studied. For systems with a nonnegative degree of homogeneity, a numeric procedure is proposed, which provides a digital representation of a homogeneous Lyapunov function. The results are illustrated by two planar examples of linear and nonlinear systems.
\end{abstract}

\section{INTRODUCTION}

Stability analysis is one of the central problems of the theories of control and dynamical systems [1], [2], [3], [4], [5], [6], [7], [8], [9]. The main approach to check the stability of a nonlinear dynamical system consists in applying the Lyapunov function method, that gives necessary and sufficient conditions for stability. The main issue with the application of this method is that there exists no procedure to design such a Lyapunov function for a generic dynamical system.

Homogeneous systems form a subclass of nonlinear systems [1], [10], that include linear systems as a special case. These systems inherit many useful properties in a generic setting from the linear case: scalability of solutions, estimation of the rate of convergence and robustness [11], [10]. A remarkable fact about homogeneous systems is that they possess homogeneous Lyapunov functions [12], [13]. However, no numeric routine has been proposed so far to construct such a Lyapunov function using homogeneity properties.

In this work, numerical tools are developed for computing an explicit homogeneous Lyapunov function. The proposed results are motivated by the important advantages of homogeneous Lyapunov functions: their construction and analysis can be performed on a sphere only, making calculations simpler. The numerical algorithms proposed here can be used for constructing homogeneous Lyapunov functions and checking stability.

The outline of this paper is as follows. Notation and preliminary results are introduced in sections II and III.

D. Efimov, R. Ushirobira and W. Perruquetti are with Inria, Non-A team Parc Scientifique de la Haute Borne, 40 avenue Halley, 59650 Villeneuve d'Ascq, France and CRIStAL (UMR-CNRS 9189), Ecole Centrale de Lille, Cité Scientifique, 59651 Villeneuve-d'Ascq, France.

J.A. Moreno is with Instituto de Ingeniería, UNAM, Coyoacán, 04510 México DF, Mexico.

This work was partially supported by ANR 15 CE23 0007 (Project Finite4SoS), the Government of Russian Federation (Grant 074-U01) and the Ministry of Education and Science of Russian Federation (Project 14.Z50.31.0031), and by HoTSMoCE Inria Associate Team.
Analysis of robustness of homogeneous Lyapunov functions is carried out in Section IV. An explicit analytic expression for homogeneous Lyapunov function is proposed in Section V. Numerical routines using those expressions to design a homogeneous Lyapunov function are presented in Section VI. The proposed approach for constructing homogeneous Lyapunov functions is illustrated by numerical examples in Section VII. Concluding remarks and discussion appear in Section VIII.

\section{NOTATION}

- $\mathbb{R}_{+}=\{x \in \mathbb{R}: x \geq 0\}$, where $\mathbb{R}$ is the set of real numbers, $\mathbb{N}$ is the set of natural numbers.

- $|\cdot|$ denotes the absolute value in $\mathbb{R},|| .||$ denotes the Euclidean norm on $\mathbb{R}^{n}$ (for $n \in \mathbb{N}$ ), $\|x\|_{\mathcal{A}}=\inf _{\xi \in \mathcal{A}}\|x-\xi\|$ is the distance from a point $x \in \mathbb{R}^{n}$ to a set $\mathcal{A} \subset \mathbb{R}^{n}$.

- A continuous function $\alpha: \mathbb{R}_{+} \rightarrow \mathbb{R}_{+}$belongs to the class $\mathcal{K}$ if $\alpha(0)=0$ and the function is strictly increasing. The function $\alpha: \mathbb{R}_{+} \rightarrow \mathbb{R}_{+}$belongs to the class $\mathcal{K}_{\infty}$ if $\alpha \in \mathcal{K}$ and it increases to infinity. A continuous function $\beta: \mathbb{R}_{+} \times \mathbb{R}_{+} \rightarrow \mathbb{R}_{+}$belongs to the class $\mathcal{K} \mathcal{L}$ if $\beta(\cdot, t) \in \mathcal{K}_{\infty}$ for each fixed $t \in \mathbb{R}_{+}$and $\lim _{t \rightarrow+\infty} \beta(s, t)=0$ for each fixed $s \in \mathbb{R}_{+}$.

- A sequence of integers $1,2, \ldots, n$ is denoted by $\overline{1, n}$.

\section{PRELIMINARIES}

Consider the following nonlinear system:

$$
\dot{x}(t)=f(x(t)), t \geq 0,
$$

where $x(t) \in \mathbb{R}^{n}$ is the state and $f: \mathbb{R}^{n} \rightarrow \mathbb{R}^{n}$ ensures forward existence and uniqueness of the system solutions at least locally, $f(0)=0$. For an initial condition $x_{0} \in \mathbb{R}^{n}$ denote the corresponding solution by $X\left(t, x_{0}\right)$ for any $t \geq 0$ for which the solution exists (the solutions are understood in the Carathéodory sense). A set $\mathcal{A} \subset \mathbb{R}^{n}$ is called forward invariant for (1) if $x_{0} \in \mathcal{A}$ implies that $X\left(t, x_{0}\right) \in \mathcal{A}$ for all $t \geq 0$.

\section{A. Stability definitions}

Following [1], [14], [15], [16], let $\Omega$ be an open neighborhood of a forward invariant set $\mathcal{A} \subset \mathbb{R}^{n}$ of (1).

Definition 1. At the set $\mathcal{A}$ the system (1) is said to be:

(a) Lyapunov stable if for any $x_{0} \in \Omega$ the solution $X\left(t, x_{0}\right)$ is defined for all $t \geq 0$, and for any $\epsilon>0$ there 
is $\delta>0$ such that for any $x_{0} \in \Omega$, if $\left\|x_{0}\right\|_{\mathcal{A}} \leq \delta$ then $\left\|X\left(t, x_{0}\right)\right\|_{\mathcal{A}} \leq \epsilon$ for all $t \geq 0$;

(b) asymptotically stable if it is Lyapunov stable and for any $\kappa>0$ and $\epsilon>0$ there exists $T(\kappa, \epsilon) \geq 0$ such that for any $x_{0} \in \Omega$, if $\left\|x_{0}\right\|_{\mathcal{A}} \leq \kappa$ then $\left\|X\left(t, x_{0}\right)\right\|_{\mathcal{A}} \leq \epsilon$ for all $t \geq T(\kappa, \epsilon)$

(c) finite-time stable if it is Lyapunov stable and finitetime converging from $\Omega$, i.e. for any $x_{0} \in \Omega$ there exists $0 \leq T<+\infty$ such that $X\left(t, x_{0}\right) \in \mathcal{A}$ for all $t \geq T$. The function $\mathcal{T}_{\mathcal{A}}\left(x_{0}\right)=\inf \left\{T \geq 0: X\left(t, x_{0}\right) \in \mathcal{A} \forall t \geq T\right\}$ is called the settling time of the system (1);

(d) fixed-time stable if it is finite-time stable and $\sup _{x_{0} \in \Omega} \mathcal{T}_{\mathcal{A}}\left(x_{0}\right)<+\infty$.

The set $\Omega$ is called the domain of stability/attraction.

If $\Omega=\mathbb{R}^{n}$, then the corresponding properties are called global Lyapunov/asymptotic/finite-time/fixed-time stability of (1) at $\mathcal{A}$.

For a $V: \mathbb{R} \rightarrow \mathbb{R}$ denote the upper Dini derivative:

$$
\dot{V}^{+}(t)=\limsup _{h \rightarrow 0^{+}} \frac{V(t+h)-V(t)}{h}, \forall t \in \mathbb{R}_{+} .
$$

If $V$ is locally Lipschitz continuous then $\dot{V}^{+}$is finite, and if $V$ is differentiable then $\dot{V}^{+}$is the usual derivative of $V$. For $V: \mathbb{R}^{n} \rightarrow \mathbb{R}$ the generalized directional derivative at $x \in \mathbb{R}^{n}$ in the direction $d \in \mathbb{R}^{n}$ is defined by:

$$
D^{+} V(x) d=\limsup _{\substack{y \rightarrow x \\ h \rightarrow 0^{+}}} \frac{V(y+h d)-V(y)}{h} .
$$

\section{B. Weighted homogeneity}

Following [1], [12], for strictly positive real numbers $r_{i}$ $(i=\overline{1, n})$ called weights and $\lambda>0$, one can define:

- the vector of weights $\mathbf{r}=\left(r_{1}, \ldots, r_{n}\right)^{T}, r_{\max }=$ $\max _{1 \leq j \leq n} r_{j}$ and $r_{\min }=\min _{1 \leq j \leq n} r_{j}$;

- the dilation matrix function $\Lambda_{r}(\lambda)=\operatorname{diag}\left(\lambda^{r_{i}}\right)_{i=1}^{n}$ (note that $\forall x \in \mathbb{R}^{n}$ and $\forall \lambda>0$ we have $\Lambda_{r}(\lambda) x=$ $\left.\left(\lambda^{r_{1}} x_{1}, \ldots, \lambda^{r_{i}} x_{i}, \ldots, \lambda^{r_{n}} x_{n}\right)^{T}\right)$;

- the $\mathbf{r}$-homogeneous norm of $x \in \mathbb{R}^{n}$ is $\|x\|_{r}=$ $\left(\sum_{i=1}^{n}\left|x_{i}\right|^{\frac{\rho}{r_{i}}}\right)^{\frac{1}{\rho}}$ for $\rho \geq r_{\max }$;

- for $s>0$ the sphere and the ball in the homogeneous norm $S_{r}(s)=\left\{x \in \mathbb{R}^{n}:\|x\|_{r}=s\right\}$ and $B_{r}(s)=\{x \in$ $\left.\mathbb{R}^{n}:\|x\|_{r} \leq s\right\}$, respectively.

Definition 2. A function $g: \mathbb{R}^{n} \rightarrow \mathbb{R}$ is $\mathbf{r}$-homogeneous of degree $\mu \in \mathbb{R}$ if $\forall x \in \mathbb{R}^{n}$ and $\forall \lambda>0$ we have:

$$
\lambda^{-\mu} g\left(\Lambda_{r}(\lambda) x\right)=g(x) .
$$

A vector field $f: \mathbb{R}^{n} \rightarrow \mathbb{R}^{n}$ is $\mathbf{r}$-homogeneous of degree $\nu \in \mathbb{R}$, with $\nu \geq-r_{\text {min }}$ if $\forall x \in \mathbb{R}^{n}$ and $\forall \lambda>0$ we have:

$$
\lambda^{-\nu} \Lambda_{r}^{-1}(\lambda) f\left(\Lambda_{r}(\lambda) x\right)=f(x),
$$

which is equivalent to the $i$-th component of $f$ being a $\mathbf{r}$-homogeneous function of degree $r_{i}+\nu$.

The system (1) is $\mathbf{r}$-homogeneous of degree $\nu$ if the vector field $f$ is $\mathbf{r}$-homogeneous of degree $\nu$.
For $\mathbf{r}$-homogeneous system (1) of degree $\nu$, the solutions also admit a kind of homogeneity as functions of time, i.e. $\forall x_{0} \in \mathbb{R}^{n}$ and $\forall t \in \mathbb{R}$ :

$$
X\left(t, \Lambda_{r}(\lambda) x_{0}\right)=\Lambda_{r}(\lambda) X\left(\lambda^{\nu} t, x_{0}\right) \quad \forall \lambda>0 .
$$

Note that $\|\cdot\|_{r}$ is $\mathbf{r}$-homogeneous function of degree 1 , and there exist $\underline{\sigma}, \bar{\sigma} \in \mathcal{K}_{\infty}$ such that

$$
\underline{\sigma}\left(\|x\|_{r}\right) \leq\|x\| \leq \bar{\sigma}\left(\|x\|_{r}\right) \quad \forall x \in \mathbb{R}^{n} .
$$

Consequently, due to this equivalence of the norms $\|\cdot\|$ and $\|\cdot\|_{r}$, the homogeneous norm can be used in Definition 1. A variant of selection of $\underline{\sigma}, \bar{\sigma}$ for a particular case considered in this work is given below:

Proposition 1. Let $r_{\max } \leq 1$ and $\rho \geq 1$, then

$$
\begin{gathered}
\underline{\sigma}(s)=\left\{\begin{array}{ll}
n^{-\frac{r_{\max }}{\rho}} s^{r_{\max }} & s \leq 1 \\
n^{-\frac{r_{\min }}{\rho}} s^{r_{\min }} & s>1
\end{array},\right. \\
\bar{\sigma}(s)=n^{\rho-\frac{1}{2}}\left\{\begin{array}{ll}
s^{r_{\min }} & s \leq 1 \\
s^{r_{\max }} & s>1
\end{array} .\right.
\end{gathered}
$$

The proofs are excluded due to space limitations.

If system (1) is $\mathbf{r}$-homogeneous with degree $\nu$, then a direct computation shows that it is also $\tilde{\mathbf{r}}$-homogeneous for $\tilde{\mathbf{r}}=r_{\max }^{-1}\left(r_{1}, \ldots, r_{n}\right)^{T}$ with degree $\tilde{\nu}=\frac{\nu}{r_{\max }}$. Therefore, the conditions of Proposition 1 can be always satisfied for any homogeneous system with $r_{\max }=\rho=1$.

Corollary 1. Let $r_{\max } \leq 1$ and $\rho \geq 1$, then $\|\cdot\|_{r}$ is locally Lipschitz continuous.

Further in this work we will always assume without loosing generality that the conditions $r_{\max } \leq 1$ and $\rho \geq 1$ are satisfied in order to use the established above continuity property of homogeneous norm $\|\cdot\|_{r}$.

\section{Time of convergence in homogeneous systems}

An important advantage of homogeneous systems is that their rate of convergence can be evaluated qualitatively based on their degree.

Theorem 1. If (1) is $\mathbf{r}$-homogeneous of degree $\nu$ and asymptotically stable at the origin, then it is

(i) globally finite-time stable at the origin if $\nu<0$;

(ii) globally exponentially stable at the origin if $\nu=0$;

(iii) globally fixed-time stable with respect to the unit ball $B_{r}(1)$ if $\nu>0$.

Following that, we can further quantify the system convergence using the definition below.

Definition 3. For $q>1$ define $T_{q}: \mathbb{R}^{n} \rightarrow \mathbb{R}_{+}$such that

$$
\left\|X\left(T_{q}\left(x_{0}\right), x_{0}\right)\right\|_{r}=q^{-1}\left\|x_{0}\right\|_{r},
$$

i.e. it is the function of contraction in q times. 
From the properties stated above, it is easy to show that $T_{q}$ is $\mathbf{r}$-homogeneous with degree $-\nu$ (the degree of homogeneity for (1)):

$$
T_{q}\left(\Lambda_{r}(\lambda) x_{0}\right)=\lambda^{-\nu} T_{q}\left(x_{0}\right), \quad \forall x_{0} \in \mathbb{R}^{n}, \forall \lambda>0 .
$$

For a $\mathbf{r}$-homogeneous system (1) of degree $\nu$, if it is asymptotically stable, then there exists a continuously differentiable and $\mathbf{r}$-homogeneous function $V: \mathbb{R}^{n} \rightarrow \mathbb{R}_{+}$of degree $\mu>-\nu$ such that for all $x \in \mathbb{R}^{n}$ :

$$
\begin{gathered}
a\|x\|_{r}^{\mu} \leq V(x) \leq b\|x\|_{r}^{\mu}, \\
D^{+} V(x) f(x) \leq-c V^{1+\frac{\nu}{\mu}}(x),
\end{gathered}
$$

for some $0<a \leq b$ and $c>0$ [12], [13]. Then for any $x_{0} \in \mathbb{R}^{n}$,

$$
\left\|X\left(t, x_{0}\right)\right\|_{r} \leq \beta\left(\left\|x_{0}\right\|_{r}, t\right) \quad \forall t \geq 0
$$

where

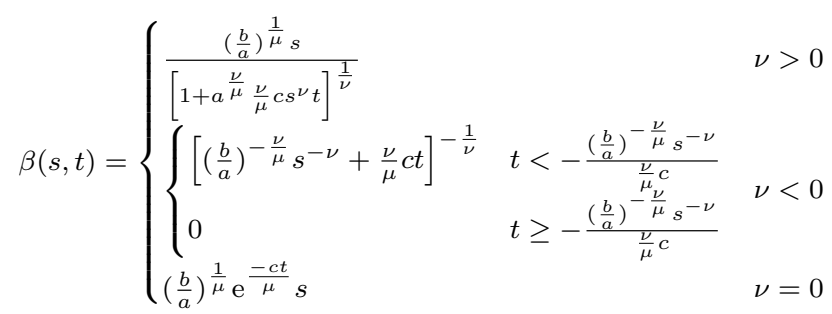

is a function from class $\mathcal{K} \mathcal{L}$, and it represents a generic parameterization of upper bound $\beta\left(\left\|x_{0}\right\|_{r}, t\right)$ for all $t \geq 0$ of $\left\|X\left(t, x_{0}\right)\right\|_{r}$ in homogeneous systems.

Definition 4. For $q>1$, define $\bar{T}_{q}: \mathbb{R}^{n} \rightarrow \mathbb{R}_{+}$such that

$$
\bar{T}_{q}\left(x_{0}\right)=\arg \inf _{T \geq 0}\left\{\left\|X\left(t, x_{0}\right)\right\|_{r} \leq q^{-1}\left\|x_{0}\right\|_{r}, \forall t \geq T\right\},
$$

i.e. it is the upper bound function of contraction in $q$ times.

Taking into account the expression of $\beta$ given in (3), we can conclude that a possible selection is $\bar{T}_{q}\left(x_{0}\right)=$ $\bar{T}_{q}\left(\left\|x_{0}\right\|_{r}\right)$ (uniform on the spheres) with

$$
\bar{T}_{q}(s)=\left\{\begin{array}{ll}
\frac{q^{\nu}\left(\frac{b}{a}\right)^{\frac{\nu}{\mu}}-1}{a^{\frac{\nu}{\mu}}} s^{-\nu} c & \nu>0 \\
\frac{\left(\frac{b}{a}\right)^{-\frac{\nu}{\mu}}-q^{\nu}}{-\frac{\nu}{\mu} c} s^{-\nu} & \nu<0 \\
\frac{\mu}{c} \ln \left[q\left(\frac{b}{a}\right)^{\frac{1}{\mu}}\right] & \nu=0
\end{array},\right.
$$

and $\bar{T}_{q}\left(x_{0}\right)$ can also be considered $\mathbf{r}$-homogeneous with degree $-\nu$ :

$$
\bar{T}_{q}\left(\Lambda_{r}(\lambda) x_{0}\right)=\lambda^{-\nu} \bar{T}_{q}\left(x_{0}\right), \forall x_{0} \in \mathbb{R}^{n}, \forall \lambda>0 .
$$

\section{ROBUSTNESS OF HOMOGENEOUS LYAPUNOV FUNCTIONS}

Let us assume that for a $\mathbf{r}$-homogeneous system (1) of degree $\nu$, there exists a locally Lipschitz continuous and $\mathbf{r}$-homogeneous function $V: \mathbb{R}^{n} \rightarrow \mathbb{R}_{+}$of degree $\mu>-\nu$, then the analysis and the verification of all properties of $V$ can be performed on the sphere $S_{r}(1)$ only. Indeed, for any $x \in \mathbb{R}^{n}$ there is $y \in S_{r}(1)$ such that $x=\Lambda_{r}\left(\|x\|_{r}\right) y$, then

$$
a\|x\|_{r}^{\mu} \leq V(x)=\|x\|_{r}^{\mu} V(y) \leq b\|x\|_{r}^{\mu},
$$

where $a=\inf _{y \in S_{r}(1)} V(y)$ and $b=\sup _{y \in S_{r}(1)} V(y)$ (same as in (2)), and

$$
D^{+} V(x) f(x)=\|x\|_{r}^{\nu+\mu} D^{+} V(y) f(y) \leq-d\|x\|_{r}^{\nu+\mu_{(4)}}
$$

where $d=-\inf _{y \in S_{r}(1)} D^{+} V(y) f(y)\left(c=b^{-1-\frac{\nu}{\mu}} d\right.$ in (2)).

Now, for such a function $V$ let us show that there is a family of locally Lipschitz continuous and $\mathbf{r}$-homogeneous Lyapunov functions $V^{\prime}: \mathbb{R}^{n} \rightarrow \mathbb{R}_{+}$of degree $\mu$ sufficiently close to $V$. To this end, denote $\varepsilon(x)=V^{\prime}(x)-V(x)$ as the difference between these functions. Clearly $\varepsilon$ is a $\mathbf{r}$-homogeneous function of degree $\mu$. We wish to formulate the restrictions on $\varepsilon$ so that $V^{\prime}(x)=V(x)+\varepsilon(x)$ would be another Lyapunov function for (1) inheriting the properties of $V$.

Proposition 2. For a locally Lipschitz continuous and $\mathbf{r}$-homogeneous function $V: \mathbb{R}^{n} \rightarrow \mathbb{R}_{+}$of degree $\mu>-\nu$, assume that the estimates (2), (4) are satisfied for constants $0<a \leq b$ and $d>0$. Let $\varepsilon: \mathbb{R}^{n} \rightarrow \mathbb{R}$ be a locally Lipschitz continuous and $\mathbf{r}$-homogeneous function of degree $\mu$ such that for all $x \in \mathbb{R}^{n}$ :

$$
-a<\underline{\varepsilon}, \quad d>\bar{\varepsilon},
$$

where $\underline{\varepsilon}=\inf _{y \in S_{r}(1)} \varepsilon(y)$ and $\bar{\varepsilon}=\sup _{y \in S_{r}(1)} D^{+} \varepsilon(y) f(y)$. Then $V^{\prime}(x)=V(x)+\varepsilon(x)$ is a locally Lipschitz continuous and $\mathbf{r}$-homogeneous Lyapunov function for (1).

\section{DESIGN OF HOMOGENEOUS LYAPUNOV FUNCTION}

In this section an explicit formula for locally Lipschitz continuous and homogeneous Lyapunov function is proposed completing the results of [12], [13], where only the existence of smooth homogeneous Lyapunov functions has been substantiated without an expression that can be used for numerical synthesis. To this end, the following hypothesis is imposed:

Assumption 1. Let (1) be asymptotically stable at the origin with a locally Lipschitz continuous and $\mathbf{r}$-homogeneous vector field $f$ of degree $\nu \geq 0$.

The possible expressions for the function $\beta \in \mathcal{K} \mathcal{L}$ are given just below (3) and the parameters of the function $\beta$, i.e. $a, b, c$ and $\mu$, are assumed to be fixed (as usual for the converse results). It is worth to stress that for the analysis in the sequel, these parameters are not related with a Lyapunov function of (1), they come from a possible parameterization of $\beta$ given after (3).

Remark 1. Recall that if the system (1) is locally Lipschitz continuous, then for any compact set of initial conditions $\mathcal{E} \subset \mathbb{R}^{n}$ and any time $0 \leqslant T<+\infty$, there exists $K_{\mathcal{E}, T} \in \mathbb{R}_{+}$ 
such that [14] (assuming that the solutions originated in $\mathcal{E}$ are defined on $[0, T]$ )

$$
\left\|X\left(t, x_{1}\right)-X\left(t, x_{2}\right)\right\| \leqslant K_{\mathcal{E}, T}\left\|x_{1}-x_{2}\right\| .
$$

Using the Lipschitz continuity of $\|\cdot\|_{r}$ established in Corollary 1 and the fact that for an asymptotically stable system $X\left(t, x_{0}\right) \in \widetilde{\mathcal{E}}$ for all $t \geq 0$ and $x_{0} \in \mathcal{E}$ for some compact $\widetilde{\mathcal{E}} \subset \mathbb{R}^{n}\left(\widetilde{\mathcal{E}}=B_{r}\left(\left(\frac{b}{a}\right)^{\frac{1}{\mu}}\right)\right.$ for $\mathcal{E}=B_{r}(1)$ according to (3)), then there exists $L_{\mathcal{E}, T} \in \mathbb{R}_{+}$such that

$$
\left|\left\|X\left(t, x_{1}\right)\right\|_{r}-\left\|X\left(t, x_{2}\right)\right\|_{r}\right| \leqslant L_{\mathcal{E}, T}\left\|x_{1}-x_{2}\right\|
$$

for all $0 \leqslant t \leqslant T$ and any $x_{1}, x_{2} \in \mathcal{E}$. These constants $K_{\mathcal{E}, T}$ and $L_{\mathcal{E}, T}$ will be used below in the proofs.

Following [17], [18], an integral converse construction of a strict Lyapunov function will be used. For the case of conventional homogeneity, such a design of Lyapunov function, with the integral on an infinite interval, was presented in [12] (Theorem 36). In this work, a more practical construction on a fixed interval of time is introduced:

Theorem 2. Let Assumption 1 be satisfied. Then there exists $q>1$ such that

$$
U(x)=\int_{0}^{\bar{T}_{q}\left(\left\|x_{0}\right\|_{r}\right)}\|X(t, x)\|_{r}^{\mu} d t,
$$

where $\bar{T}_{q}\left(\left\|x_{0}\right\|_{r}\right)$ is the upper bound function of contraction in $q$ times, for $\mu>\max \left\{1, \nu+\nu^{2}\right\}$ is a locally Lipschitz continuous and $\mathbf{r}$-homogeneous of degree $\mu-\nu$ function such that

$$
\begin{gathered}
T_{q}^{\min } q^{-\mu}\|x\|_{r}^{\mu-\nu} \leqslant U(x) \leqslant \bar{T}_{q}(1) \frac{b}{a}\|x\|_{r}^{\mu-\nu}, \forall x \in \mathbb{R}^{n}, \\
D^{+} U(x) f(x)<0 \quad \text { for a.a. } x \in \mathbb{R}^{n} \backslash\{0\},
\end{gathered}
$$

where $T_{q}^{\min }=\inf _{x_{0} \in S_{r}(1)} T_{q}\left(x_{0}\right)$.

\section{NUMERIC DESIGN}

Let us consider how the Lyapunov function proposed in Theorem 2 can be constructed numerically.

For a given fixed discretization step $h>0$, let $X_{h}\left(t_{i}, x_{0}\right)$ denote an approximation at instants $t_{i}=i h, i \geq 0$ of the solution $X\left(t, x_{0}\right)$ of (1) using Euler method [19] (any other discretization method can also be used).

\section{A. Pointwise calculation}

For $N>0$, let $\xi_{j} \in S_{r}(1)$ with $j=\overline{1, N}$ form a uniform grid on the unit sphere $S_{r}(1)$, and let us introduce into consideration the following variables:

$$
\begin{gathered}
U_{j}^{h}=h \sum_{i=0}^{J}\left\|X_{h}\left(t_{i}, \xi_{j}\right)\right\|_{r}^{\mu}, \\
J=\tau \underset{i \geq J}{\arg \sup _{i \leq j \leq N}} \sup _{1 \leq X_{h}\left(t_{i}, \xi_{j}\right) \|_{r} \leq q^{-1},}
\end{gathered}
$$

where $\tau>1$ is a tuning parameter.
Corollary 2. Let Assumption 1 be satisfied. Then there exist $q>1, N>0$ and $h>0$ such that for any $\tau>0$ and $\mu>\max \left\{1, \nu+\nu^{2}\right\}$ :

$$
U_{j}^{h}=U\left(\xi_{j}\right) \quad \forall j=\overline{1, N},
$$

where $U: \mathbb{R}^{n} \rightarrow \mathbb{R}_{+}$is a locally Lipschitz continuous and $\mathbf{r}$-homogeneous of degree $\mu-\nu$ Lyapunov function for the system (1).

\section{B. Interpolation}

Denote a weighted product of all elements of the state vector by

$$
p(x, \alpha)=\prod_{i=1}^{n}\left\lceil x_{i}\right\rfloor^{\alpha_{i}},
$$

where $x=\left[x_{1} \ldots x_{n}\right]^{T},\left\lceil x_{i}\right\rfloor^{\alpha_{i}}=\left|x_{i}\right|^{\alpha_{i}} \operatorname{sign}\left(x_{i}\right)$ and $\alpha=$ $\left[\alpha_{1}, \ldots, \alpha_{n}\right]^{T} \in \mathbb{R}_{+}^{n}$, and let

$$
\begin{aligned}
\mathcal{P}_{\rho}= & \left\{p(x, \alpha), \alpha \in \mathbb{R}_{+}^{n}: p\left(\Lambda_{r}(\lambda) x, \alpha\right)=\lambda^{\rho} p(x, \alpha)\right. \\
& \left.\forall \lambda>0, \forall x \in \mathbb{R}^{n}\right\}
\end{aligned}
$$

be the set of all possible $\mathbf{r}$-homogeneous products of degree $\rho$. For an integer $s \in[1, N]$ consider a polynomial function

$$
u\left(x, \theta_{j}\right)=\sum_{z=1}^{s} \theta_{z}^{j} p_{z}(x)
$$

where $p_{z} \in \mathcal{P}_{\mu-\nu}$ and $\theta_{j}=\left[\theta_{1}^{j}, \ldots, \theta_{s}^{j}\right]^{T} \in \mathbb{R}^{s}$ is the vector of parameters, then the values of $U$ between $U_{j}^{h}$ and $U_{j+1}^{h}$, or on the interval $\left[\xi_{j}, \xi_{j+1}\right]$ on the sphere $S_{r}(1)$ (here we assume that $U_{N+1}^{h}=U_{1}^{h}$ and $\xi_{N+1}=\xi_{1}$ ), can be interpolated for all $x \in\left[\xi_{j}, \xi_{j+1}\right]$ using the $\mathbf{r}$-homogeneous of degree $\mu-\nu$ polynomial $u\left(x, \theta_{j}\right)$. In this case the vector $\theta_{j}$ can be calculated as a solution of the system of linear equations:

$$
U_{k}^{h}=u\left(\xi_{k}, \theta_{j}\right) \quad k=j-\frac{s}{2}+1, \ldots, j+\frac{s}{2}
$$

for an even $s$ (if $s$ is odd then $k=j-\frac{s-1}{2}+1, \ldots, j+\frac{s+1}{2}$ ). If each polynomial $u\left(x, \theta_{j}\right)$ is continuously differentiable on $\left[\xi_{j}, \xi_{j+1}\right]$, i.e. when all powers in $\alpha$ are higher than one, then the following equations can be substituted in the previous system in order to ensure that the differentiability is preserved on $S_{r}(1)$ :

$$
\left.\frac{\partial u\left(x, \theta_{j}\right)}{\partial x}\right|_{x=\xi_{j+1}}=\left.\frac{\partial u\left(x, \theta_{j+1}\right)}{\partial x}\right|_{x=\xi_{j+1}} .
$$

More compactly this system of equations can be presented in the form

$$
\Upsilon_{N}=\Pi_{N} \Theta_{N}
$$

where $\Theta_{N}=\left[\theta_{1}^{T} \ldots \theta_{N}^{T}\right]^{T} \in \mathbb{R}^{N s}$ is the vector of all parameters, $\Upsilon_{N} \in \mathbb{R}^{N s}$ is the vector composed by the corresponding values of $U_{k}^{h}$, and $\Pi_{N} \in \mathbb{R}^{N s \times N s}$ is a matrix of values of polynomials $p_{z}\left(\xi_{k}\right)$ (and their partial derivatives).

Assumption 2. There exists $\varrho>0$ such that for any $N>0$

$$
\left\|\Pi_{N}^{-1}\right\|_{2} \leq \frac{\varrho}{N}
$$



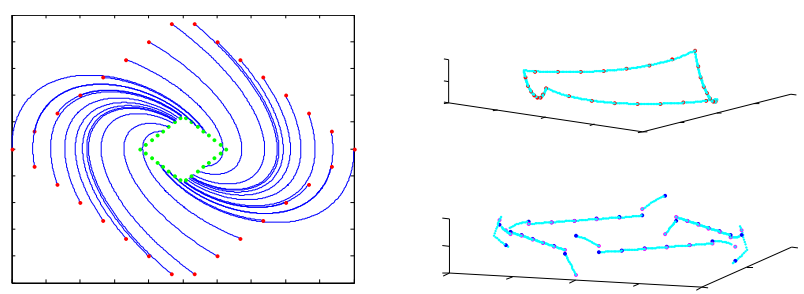

Figure 1. Results of calculations for linear system

where $\|\cdot\|_{2}$ is the induced matrix norm.

This assumption can be ensured for any $N$ by a careful selection of polynomials $p_{z} \in \mathcal{P}_{\mu-\nu}$ in (5). Then for a sufficiently big value of $N$ (for a sufficiently dense grid on $S_{r}(1)$ ) the derivative of this polynomial can be made sufficiently small, which implies that the result of Proposition 2 can be applied and the conditions on $\underline{\varepsilon}$ and $\bar{\varepsilon}$ are satisfied:

Theorem 3. Let assumptions 1 and 2 be satisfied. Then there exist $q>1, N>0$ and $h>0$ such that for any $\tau>0, s>0$ and $\mu>\max \left\{1, \nu+\nu^{2}, \nu+1\right\}$, a locally Lipschitz continuous and $\mathbf{r}$-homogeneous of degree $\mu-\nu$ Lyapunov function for the system (1) can be found on $S_{r}(1)$ in the form (5), where the parameters $\theta_{j}$ are solutions of (6).

\section{EXAMPLES}

For simplicity of presentation, the case $n=2$ is investigated in this section, and the following expressions and values of parameters are used for computation of a homogeneous Lyapunov function:

$$
\begin{gathered}
\mu=\max \left\{\nu+1, \max \left\{1, \nu+\nu^{2}\right\}, \nu+\sum_{i=1}^{n} r_{i}\right\}+1, \\
N=30, q=4, \tau=1.25, h=0.01 \\
\alpha=\left[\begin{array}{cccc}
\frac{\mu-\nu}{r_{1}} & 0 & 1 & \frac{\mu-\nu-r_{2}}{r_{1}} \\
0 & \frac{\mu-\nu}{r_{2}} & \frac{\mu-\nu-r_{1}}{r_{2}} & 1
\end{array}\right] .
\end{gathered}
$$

\section{A. Linear system}

Consider (1) with

$$
f(x)=\left[\begin{array}{c}
x_{2} \\
-x_{1}-x_{2}
\end{array}\right]
$$

for $x \in \mathbb{R}^{2}$, then $\nu=0$ for $\mathbf{r}=\left[\begin{array}{ll}1 & 1\end{array}\right]$. The results of simulation are shown in Fig. 1. The state trajectories, as well as the homogeneous norm levels 1 and $q^{-1}$, are presented in Fig. 1,a. The obtained values of $U$ are plotted in Fig. 1,b (the red points represent $U_{j}^{h}$ ), the numeric derivative of $U$ is given in Fig. 1,c (the blue and magenta points represent the values of derivative at $\xi_{j}$ for the neighboring approximations with $\theta_{j}$ and $\left.\theta_{j-1}\right)$. As we can conclude from these results the obtained values correspond to a Lyapunov function, and it is homogeneous by construction.
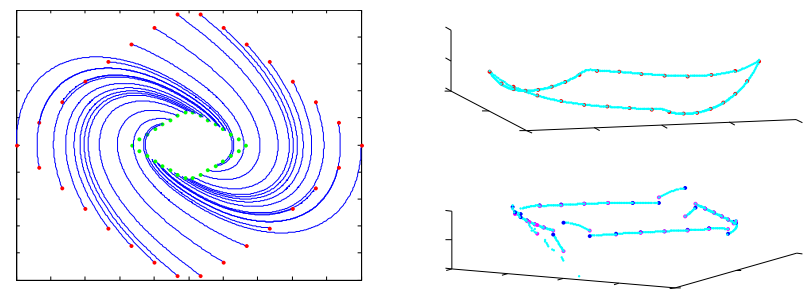

Figure 2. Results of calculations for nonlinear system

\section{B. Nonlinear system}

Consider (1) with

$$
f(x)=\left[\begin{array}{c}
x_{2} \\
-\left\lceil x_{1}\right\rfloor^{3}-\left\lceil x_{2}\right\rfloor^{1.5}
\end{array}\right]
$$

for $x \in \mathbb{R}^{2}$, then $\nu=0.5$ for $\mathbf{r}=\left[\begin{array}{ll}0.5 & 1\end{array}\right]$. The results of simulation are shown in Fig. 2 (trajectories in Fig. 2,a, the values of $U$ in Fig. 2,b and the derivative in Fig. 2,c). The results of these calculations also confirm our theoretical findings.

\section{CONCLUSION}

The problem of constructing a homogeneous Lyapunov function for an asymptotically stable homogeneous system is revisited. First, for systems with nonnegative degree of homogeneity, an expression of a homogeneous Lyapunov function is proposed, which depends explicitly on the system solutions (on finite intervals of time). Second, a procedure is presented, which under some technical assumptions ensures that a homogeneous Lyapunov function can be numerically constructed. The results are illustrated by simulations for linear and nonlinear cases. The proposed numeric design of Lyapunov functions can be used for verification of stability of homogeneous systems. Future directions of research include extensions of the approach for the cases of negative degree and discontinuous dynamics.

\section{REFERENCES}

[1] A. Bacciotti and L. Rosier, Liapunov Functions and Stability in Control Theory, ser. Lecture Notes in Control and Inform. Sci. Berlin: Springer, 2001, vol. 267.

[2] N. Bhatia and G. Szegö, Dynamical Systems: Stability Theory and Applications. Berlin: Springer-Verlag, 1967.

[3] N. Chetaev, The Stability of Motion. New York: Pergamon Press, 1961, (English translation).

[4] A. Fradkov and A. Pogromsky, Introduction to oscillations and chaos. Singapore: World Scientific, 1998.

[5] W. Hahn, Stability of Motion. Berlin: Springer, 1967.

[6] A. Isidori, Nonlinear control systems: An Introduction, 2nd ed. Berlin: Springer-Verlag, 1989.

[7] N. Krasovskii, Stability of Motion: Applications of Lyapunov's Second Method to Differential Systems and Equations With Delay. Stanford University Press, 1963.

[8] A. Lyapunov, The general problem of the stability of motion. London: Taylor \& Francis, 1992, translated by A. T. Fuller.

[9] R. Sepulchre, M. Jankovic, and P. Kokotović, Constructive nonlinear control. NY: Springer-Verlag, 1997.

[10] E. Bernuau, D. Efimov, W. Perruquetti, and A. Polyakov, "On homogeneity and its application in sliding mode control," Journal of the Franklin Institute, vol. 351, no. 4, pp. 1866-1901, 2014. 
[11] E. Bernuau, A. Polyakov, D. Efimov, and W. Perruquetti, "Verification of ISS, iISS and IOSS properties applying weighted homogeneity," Systems \& Control Letters, vol. 62, no. 12, pp. 1159-1167, 2013.

[12] V. Zubov, Methods of A.M. Lyapunov and Their Application, P. Noordhoff, Ed. Stechert-Hafner Service Agency, 1964.

[13] L. Rosier, "Homogeneous lyapunov function for homogeneous continuous vector field," Systems \& Control Lett., vol. 19, pp. 467-473, 1992.

[14] H. Khalil, Nonlinear Systems, 3rd ed. Upper Saddle River, New Jersey: Prentice Hall, 2002.

[15] Y. Lin, E. Sontag, and Y. Wang, "A smooth converse Lyapunov theorem for robust stability," SIAM J. Control Optimization, vol. 34, pp. 124160, 1996.

[16] A. Polyakov, "Nonlinear feedback design for fixed-time stabilization of linear control systems," IEEE Transactions on Automatic Control, vol. 57, no. 8, pp. 2106-2110, 2012.

[17] J. Massera, "On Liapounoff's conditions of stability," Annals of Mathematics, vol. 50, pp. 705-721, 1949.

[18] J. Kurzweil, "On the reversibility of the first theorem of Lyapunov concerning the stability of motion," Czechoslovak Mathematical Journal, vol. 5, pp. 382-398, 1955.

[19] J. C. Butcher, Numerical Methods for Ordinary Differential Equations. New York: John Wiley \& Sons, 2003. 